\title{
Una estrategia poco utilizada en el cuidado de pacientes con Enfermedad Renal Crónica: la educación en grupo y multidisciplinar de pacientes y sus familiares
}

Ana Isabel Aguilera Flórez ${ }^{1}$ - Mario Prieto Velasco ${ }^{2}$ - Luis González Romero ${ }^{3}$ - Beatriz Abad Toral ${ }^{1}$ - Elisa Martínez Crespo ${ }^{1}$ - Israel Robles del Rio ${ }^{1}$ - Esperanza Gutiérrez Gutiérrez ${ }^{4}$ - Alicia Calleja Fernández ${ }^{5}$ - Pilar de Boso Serrano ${ }^{6}$

${ }^{1}$ Enfermera

${ }^{2}$ Nefrólogo

${ }^{3}$ Fisioterapeuta y enfermero

${ }^{4}$ Farmacéutica

${ }^{5}$ Dietista-Nutricionista. Complejo Asistencial Universitario de León

${ }^{6}$ Psicóloga. Centro de Diálisis ASHDO León

\section{Resumen}

Los equipos multidisciplinares, son una estrategia sugerida hace ya más de un decenio por grupos canadienses. Los pacientes tratados en un ámbito multidisciplinar demuestran un mejor conocimiento de la enfermedad renal crónica y mejores parámetros bioquímicos.

Los objetivos fueron, aumentar los conocimientos, en nuestros pacientes con enfermedad renal crónica avanzada y su familia, sobre su enfermedad, su correcto cuidado y sobre el tratamiento renal sustitutivo, a través de la educación en grupo. Valorar si la información aumenta el nivel de ansiedad de los pacientes. Valorar el grado de satisfacción con la intervención educación en grupo.

Estudio retrospectivo que contó con 19 pacientes, asistentes a los dos cursos. Se realizaron dos cuestionarios al inicio y al final del curso sobre nivel de conocimientos y el nivel de ansiedad (Inventario de Ansiedad Estado-Rasgo). Al final de cada edición, se realizó un cuestionario de evaluación. Las variables estudiadas fueron el sexo, edad, nivel de estudios y asistencia de un familiar.

El nivel de conocimientos alcanzó una diferencia estadísticamente significativa entre ambos cuestionarios (inicial-final) $(p=0,013)$. Los resultados obtenidos en la evaluación del nivel de ansiedad mostraron un ligero incremento en la ansiedad. El cuestionario de satisfacción mostró una valoración muy positiva de la intervención.

Podemos concluir, que la mayoría de los pacientes han aumentado sus conocimientos sobre enfermedad renal crónica avanzada y técnicas de sustitución, han demostrado una alta satisfacción con la experiencia de esta intervención y la mayoría han experimentado un ligero aumento de ansiedad. 
A little-used strategy in caring for patients with chronic kidney disease: multidisciplinary education of patients and their relatives

\section{Abstract}

Multidisciplinary teams are a strategy that was suggested more than ten years ago by Canadian groups. Patients treated in an multidisciplinary environment show better knowledge of CKD and better biochemical parameters.

The goals were to increase knowledge in our patients with ACKD and their family about their illness, correct care and renal replacement therapy, through group education, to evaluate whether the information increased the patients' level of anxiety and to evaluate the degree of satisfaction with the group education.

This is a retrospective study carried out with 19 patients, who attended both courses. Two questionnaires were completed at the start and end of the course on the level of knowledge and level of anxiety (State-Trait Anxiety Inventory STAI). At the end of each course, an assessment questionnaire was completed. The variables studied were sex, age, level of education and presence of a relative.

The level of knowledge reached a statistically significant difference between the two questionnaires (start-end) $(p=0.013)$. The results obtained in the evaluation of the level of anxiety showed a slight increase in anxiety. The satisfaction questionnaire showed a very positive evaluation of the action.

We can conclude that most of the patients have increased their knowledge of ACKD and replacement techniques, have shown a high degree of satisfaction with the experience of this intervention and most have experienced a slight increase in anxiety.

\section{KEY WORDS:}

\section{- MULTIDISCIPLINARY TEAM}

- GROUP EDUCATION

- ADVANCED CHRONIC KIDNEY DISEASE

- RENAL REPLACEMENT THERAPY

\section{Introducción}

La enfermedad renal crónica (ERC) se define por la disminución del filtrado glomerular por debajo de 60 $\mathrm{ml} / \mathrm{min} / 1,73 \mathrm{~m}^{2}$ de SC y/o proteinuria y/o alteración del sedimento $\mathrm{y} / 0$ alteraciones en el diagnóstico por la imagen de los riñones. Se clasifica en 5 estadios, dependiendo del grado de pérdida de función renal. Se le llama ERC avanzada (ERCA) a los estadios 4-5 de la clasificación; es decir, filtrado glomerular $<30 \mathrm{ml} / \mathrm{min}^{1}$. La prevalencia de ERC en España está en el 6,8\% y la prevalencia de ERCA está en torno a 3 personas por cada mil habitantes; aunque en mayores de 64 años, está por encima de 1 por cada 100 habitantes ${ }^{2}$. Hay un número creciente de trabajos que acumulan evidencia a favor de que el envío temprano del paciente a la consulta de nefrología, especialmente en un contexto de unidades ERCA con un equipo multidisciplinar (EMD), facilita el acceso a una información más detallada y balanceada, proporciona un cuidado más eficiente, efectivo y global tanto de los pacientes como de sus familiares, enlentece la progresión de la ERC, disminuye la comorbilidad y las complicaciones de la ERC, promueve la elección informada de la técnica de tratamiento y prepara a los pacientes para el autocuidado y la terapia domiciliaria si es la elección realizada. Todos los trabajadores de la salud en las distintas áreas debemos contribuir a estos estándares de cuidado 3 . Según se recoge en la guía SEN, ERCA y Prediálisis, una asistencia óptima en la fase ERCA, debe contemplar: 1) detección precoz de la ERC progresiva; 2 ) intervenciones para retardar su progresión; 3) prevenir las complicaciones urémicas; 4) atenuar las condiciones comórbidas asociadas; 5) adecuar la preparación para el tratamiento renal sustitutivo e iniciarlo en el momento adecuado y de forma programada, 6) la alternativa de futuro son los equipos multidisciplinares de manejo de la ERC avanzada.

El tratamiento multidisciplinar presenta los siguientes objetivos: 1) asistencia; 2) información; 3) formación; 4) educación para integrar a los pacientes de manera activa en todo el proceso. Los pacientes con un bajo nivel de conocimientos sobre salud tienen un nivel de salud menor, mayor número de hospitalizaciones y es menos probable que cumplan el tratamiento prescrito y los planes de autocuidado, así como que tengan más errores en el cumplimiento del tratamiento y hagan 
menos uso de las estrategias de prevención ${ }^{4}$. Además, se debe implicar, siempre que sea posible, a un miembro de la familia más próxima, porque tal cómo está recogido en la literatura, en las personas cercanas al paciente, se reconocen al menos dos áreas principales de impacto a) tanto la DP como la HD tienen un papel de interferencia en la vida social de los familiares; y en muchas ocasiones la estructura organizativa de la semana viene determinada por las sesiones de diálisis y b) muchos pacientes se vuelven más frágiles y funcionalmente más dependientes, precisando que las familias les presten un apoyo físico mayor ${ }^{5}$. Por tanto, la familia también debe ser informada para que opine y sea escuchada en todo el proceso y contribuya al éxito del tratamiento.

Frente a la habilidad individual del nefrólogo para atender los múltiples y complejos aspectos del cuidado de estos pacientes están los EMD, estrategia sugerida hace ya más de un decenio por grupos canadienses. Los pacientes tratados en un ámbito EMD demuestran un mejor conocimiento de la ERC y mejores parámetros bioquímicos ${ }^{6}$.

En nuestra consulta ERCA, con el objetivo de implicar al paciente y su familia en la toma de decisiones y el cumplimiento del tratamiento, estamos utilizando distintos formatos de trasmitir información, entre ellos la consulta específica de enfermería nefrológica con consultas repetidas (3-5) para la información de opciones, dieta. etc..; las herramientas de ayuda en la toma de decisiones; etc...Y hace más de un año nos propusimos utilizar, además, una estrategia practicada en otros campos de la medicina, pero poco utilizada en ERCA: la educación en grupo a pacientes y sus familiares impartida por un equipo multidisciplinar de profesionales sanitarios.

Los objetivos que nos hemos planteado en este trabajo son:

\section{Objetivo principal:}

- Valorar si la educación grupal impartida por un equipo multidisciplinar de profesionales, aumenta los conocimientos en nuestros pacientes con ERCA y su familia sobre su enfermedad, sobre el tratamiento renal sustitutivo y su correcto cuidado (farmacológico, higiénico y mental).

\section{Objetivos secundarios}

- Valorar si la información aumenta el nivel de ansiedad de los pacientes.

- Valorar el grado de satisfacción con la intervención de la educación en grupo.

\section{Material y método}

El equipo multidisciplinar fue constituido en enero de 2010 formado por 11 profesionales de diferentes ámbitos sanitarios que participan en el cuidado y en la educación sanitaria del paciente renal, se denominó "Escuela ERCA". En cada edición de la escuela, son organizadas 7 jornadas con una frecuencia quincenal y una duración de hora y media, en las que se imparten 3 temas de los diferentes ámbitos de interés en la enfermedad renal. Cada tema está dividido en dos partes: 20 minutos de teoría y 10 minutos de debate, aunque los temas más controvertidos conllevan más tiempo del previsto.

Los pacientes que acuden a la "Escuela ERCA" han sido seleccionados previamente en la consulta de $\mathrm{Ne}$ frología ERCA, y todos han pasado por un proceso de educación individualizada.

Los criterios de inclusión son: MDRD $<20 \mathrm{ml} / \mathrm{min} / 1.73 \mathrm{~m}^{2}$ y voluntad del paciente para formar parte del grupo. Cada grupo está formado por diez pacientes, con la posibilidad de ser acompañado por un familiar, con el fin de mejorar, de forma simultánea, la educación del paciente y cuidador.

Los temas que se tratan en la "Escuela ERCA" son: EI riñón y su funcionamiento, modalidades de tratamiento sustitutivo (HD,DP,TX), dieta, medicación, vacunación, anemia, actividad física, aspectos psicológicos , experiencia personal de los pacientes .

Los temas tratados, son expuestos por los diferentes profesionales sanitarios integrantes del grupo, desde un enfoque activo y participativo. Para las exposiciones se utilizaron presentaciones Power Point y coloquios entre los participantes.

El grupo de profesionales está coordinado por un nefrólogo y una enfermera de la consulta de ERCA. 
Forman parte de él: tres enfermeros, dos nefrólogos, una dietista-nutricionista, una farmacéutica, una psicóloga y un fisioterapeuta.

Las funciones y los objetivos de cada uno de los integrantes del grupo son:

- Coordinadores: Son el nexo de unión entre pacientes y profesionales de la escuela. Están presentes en todas las sesiones. Se encargan de la selección de los pacientes y coordinar a los integrantes del grupo. Además, elaboran en consenso el calendario de actividad y evalúan las diferentes actividades.

- Nefrólogos: Dan información y formación sobre la enfermedad renal, su tratamiento y complicaciones más importantes.

- Enfermeras: Su aportación consiste en aumentar los conocimientos del paciente y familia, facilitando que desarrollen capacidades que les permitan tomar decisiones conscientes y autónomas sobre temas relacionados con las modalidades de tratamiento sustitutivo renal y como pueden afectar a su estilo de vida, para que puedan alcanzar un grado de conocimiento que les ayude a tomar una decisión en cuanto a la modalidad de tratamiento sustitutivo renal que quieran hacer, formarles sobre el manejo correcto de la Eritropoyetina(EP0), programa de vacunación encaminado a prevenir la aparición de enfermedades víricas que puedan llegar a ser graves, consejos dietéticos en combinación con la DietistaNutricionista con el fin de conseguir que los pacientes adquieran unos hábitos alimenticios adecuados que les permitan mejorar su calidad de vida.

- Psicóloga: Su función es ayudarles a mejorar el afrontamiento de la enfermedad, así como, valorar la repercusión que tiene la información en el nivel de ansiedad de los pacientes que acuden a la "Escuela ERCA". Su objetivo será darles a conocer las alteraciones psicológicas más frecuentes en el paciente renal para que puedan identificarlas y proporcionarles conocimientos útiles sobre aquellos aspectos que favorezcan la adaptación, el afrontamiento y la calidad de vida. Asimismo orientará y recomendará pautas de actuación a las familias para que puedan comprender su situación emocional y prestarles un apoyo más eficaz.
- Farmacéutica: Su función es ayudar a los pacientes a entender los medicamentos que toman, la razón por la que se los han prescrito y cómo deben tomarlos. El objetivo que se quiere conseguir es aumentar sus conocimientos sobre la importancia de los fármacos en la enfermedad renal, para qué sirven y cuál es la mejor manera de administrarlos, y que reconozcan aquellos fármacos que deben evitarse 0 aquellos con los que es necesario tener más precauciones en la enfermedad renal.

- Dietista-Nutricionista: Su función principal será educar nutricionalmente en temas relacionados con los alimentos, la hidratación, técnicas culinarias, así como medidas higiénico-dietéticas en la vida diaria. El objetivo principal de esta temática será educar nutricionalmente al paciente renal dándole las herramientas necesarias para que su alimentación sea sana y equilibrada, y que además esté adaptada al estadio de su enfermedad renal, así como al tratamiento renal sustitutivo.

- Fisioterapeuta: Su función es, aportar los conocimientos del campo de la fisioterapia, que establece la relación necesaria entre los conceptos de salud y ejercicio, cumpliendo la estrategia mundial de la OMS sobre régimen alimentario, actividad física y salud. Su objetivo es incidir en los beneficios que la actividad física regular tiene para la salud, reforzando hábitos saludables. Describir las características particulares de la actividad física adaptada al paciente nefrológico. Realizar una tabla de ejercicios adaptados, que fomenten la participación activa, resolviendo dudas sobre el tema tratado.

- Pacientes mentores: Su función es relatar su experiencia personal como paciente renal, cómo se enfrentan día a día a la enfermedad y como les ha afectado a su estilo de vida. Su objetivo, disminuir el temor ante la enfermedad renal y su tratamiento. Acuden tres pacientes, uno por cada modalidad de tratamiento sustitutivo renal (hemodiálisis, diálisis peritoneal y trasplante).

Con el fin de valorar la eficacia del equipo multidisciplinar en la intervención educación grupal, nos planteamos medir distintos parámetros:

Nivel de conocimientos, para ello y previo al inicio de las sesiones grupales fue entregado a cada uno de 
los participantes un cuestionario de conocimientos con 12 preguntas multirrespuesta sobre diferentes temas: generalidades de la enfermedad renal, alimentación, fármacos y tratamiento renal sustitutivo. Una vez finalizada las sesiones grupales, fue realizado de nuevo el mismo cuestionario para evaluar los conocimientos adquiridos. Los datos fueron introducidos en un documento de Excel y analizados estadísticamente con el programa SPSS 15.0. Los datos cuantitativos figuran como media y los cualitativos como frecuencias. Se empleó la "t de student" para la comparación entre medias. Se estimó una diferencia estadísticamente significativa un valor $p<0,05$.

Para medir el nivel de ansiedad se les paso al inicio y al final de las sesiones grupales el Inventario de Ansiedad Estado-Rasgo STAI ${ }^{7}$ es un inventario de carácter psicométrico que consta de dos escalas de autoevaluación que miden facetas diferentes, pero relacionadas de la ansiedad: el estado y el rasgo. La escala de estado se responde informando sobre la intensidad con que aparecen en un momento concreto sentimientos o sensaciones de ansiedad (nada, algo, bastante, mucho). La escala de rasgo debe responder indicando como se siente habitualmente (casi nunca, a veces, a menudo, casi siempre).

Para evaluar el grado de satisfacción con la intervención de enseñanza en grupo, se pasó un cuestionario de evaluación, que contenía 15 preguntas: 9 que debían ser valoradas de 1 a 5 , siendo el valor 1 muy negativo y el 5 muy positivo, 2 preguntas como insuficiente, adecuada o excesiva y 3 preguntas como respuesta afirmativa o negativa.

\section{Resultados}

Se han realizado dos cursos con un total de 19 pacientes $(n=19)$, de los cuales el $73,68 \%(n=14)$ eran hombres y el $26,31 \%(n=5)$ eran mujeres, con una edad media de 58,1 años y un rango entre (32-76). De estos pacientes, el 73,68\% $(n=14)$ fueron acompañados de un familiar (en 8 pacientes el acompañante era su esposa/o, en 3 hijos, en 2 un hermano y en 1 su padre) y el 26,31 ( $n=5)$ acudieron solos. En cuanto al nivel de estudios, el $26,3 \%$ $(n=5)$ tenían estudios universitarios, el 36,8\% $(n=7)$ estudios de grado medio y el $36,8 \%(n=7)$ estudios básicos. La media del filtrado glomerular que los pacientes presentaban al comienzo del curso era de $12,57 \mathrm{ml} / \mathrm{min}$, con un rango entre $(8-16)$. La etiología de la enfermedad renal y el origen de la nefropatía fue diverso: diabetes 10,5\% $(n=2)$, Glomerulares $31,5 \%(n=6)$, Hereditarias $10,5 \%(n=2)$, Vasculares $15,80(n=3)$, Intersticiales $15,80(n=3)$, No filiadas $15,80(n=3)$. En cuanto al nivel de conocimientos fueron analizados 15 cuestionarios, cuatro pacientes no realizaron el cuestionario final, por faltar a la última sesión del grupo. La nota media inicial fue de 7,2 puntos y de 9,3 puntos al final, con una diferencia estadísticamente significativa entre ambas $(p=0,013)$. Como se observa en el gráfico, se produce un incremento en el porcentaje de aciertos a excepción de las preguntas 3 (¿Qué alimentos son ricos en fósforo?) y 7 (¿Qué parámetros son importantes para cuantificar la función renal?). (Figural)

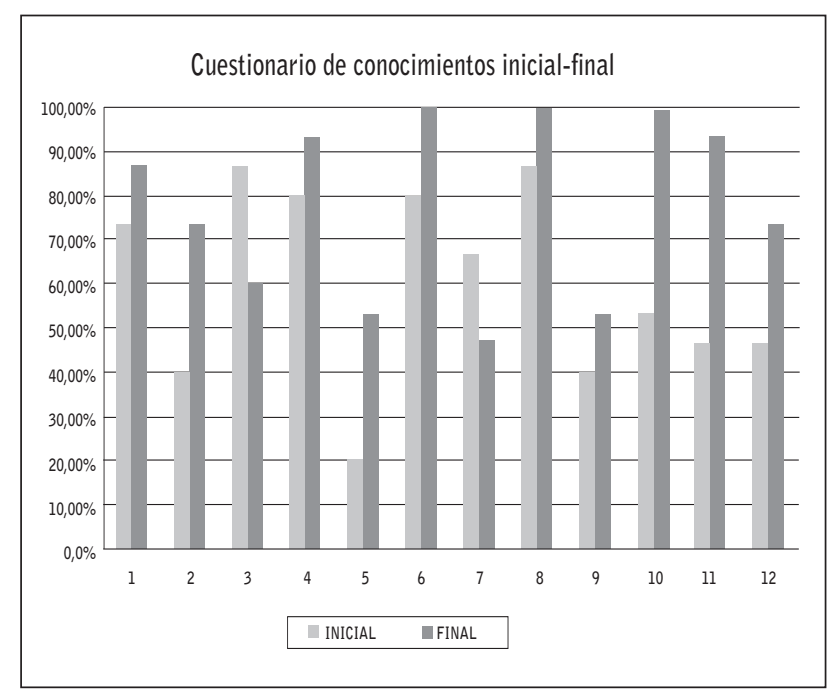

Figura 1. Valoración del cuestionario de conocimientos inicial-final.

En la $2^{\text {a }}$ edición de la Escuela de ERCA, con el fin de medir el nivel de ansiedad que presentaban los pacientes antes y después de asistir al curso, se aplicó el test "Inventario de Ansiedad Estado-Rasgo STAI"(6) al inicio y al final. De los 11 pacientes evaluados inicialmente sólo 8 realizaron el cuestionario final. Los resultados obtenidos evidenciaron que el $37,5 \% \quad(n=3)$ no mostraron variación en sus niveles de ansiedad, el $50 \%(n=4)$ mostraron un ligero incremento en la ansiedad y el $12,5 \%(n=1)$ reflejó una disminución en sus niveles de ansiedad después de asistir a la "Escuela ERCA". (Figura 2). 
[ A. I. Aguilera Flórez, et al ]

Una estrategia poco utilizada en el cuidado de pacientes con Enfermedad Renal Crónica: la educación en grupo y multidisciplinar de pacientes y sus familiares

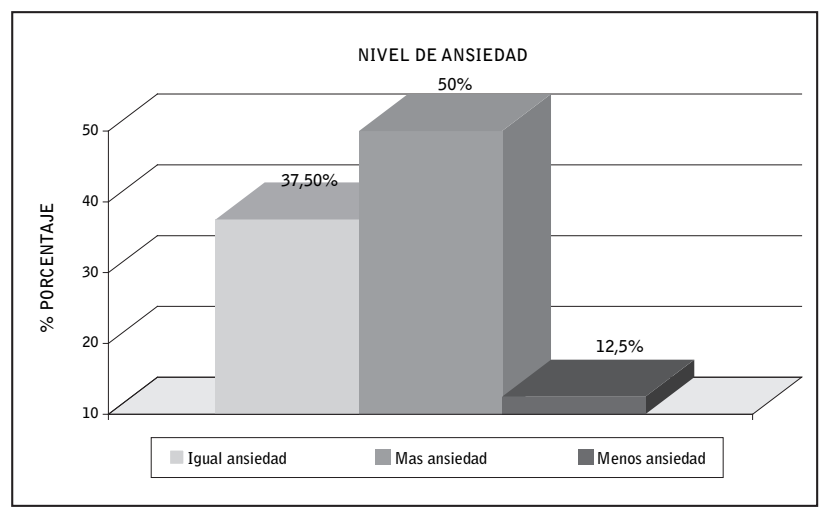

Figura 2. Nivel de ansiedad
El cuestionario para valorar el grado de satisfacción con la "Escuela ERCA" fue respondido por 18 pacientes en las dos ediciones. Los resultados figuran en la Tabla como frecuencias (número de respuestas) y la valoración como media (Desviación Estándar). Además, se incluyó un apartado de sugerencias donde fueron incluidas las felicitaciones por el transcurso de la escuela y tan sólo un paciente, indicó que el aumentar los conocimientos sobre su patología ha aumentado su preocupación por su enfermedad y no considera positiva la actividad. (Tablal).

\begin{tabular}{|c|c|c|c|c|c|c|}
\hline \multicolumn{7}{|l|}{ Valoración de las preguntas de la 1-9 } \\
\hline & 1 & 2 & 3 & 4 & 5 & Media (DS) \\
\hline Valoración general & 0 & 0 & 0 & $11,1 \%(2)$ & $88,9 \%(16)$ & $4,9(0,3)$ \\
\hline Utilidad de los temas & 0 & 0 & 0 & $23,5 \%(4)$ & $76,5 \%(13)$ & $4,8(0,4)$ \\
\hline Profundidad de los temas & 0 & 0 & $5,6 \%(1)$ & $11,1 \%(2)$ & $83,3 \%(15)$ & $4,8(0,5)$ \\
\hline Capacidad de los ponentes para mantener la atención & 0 & 0 & 0 & $11,1 \%(2)$ & $88,9 \%(16)$ & $4,9(0,3)$ \\
\hline Capacidad de los ponentes para fomentar la participación & 0 & 0 & $11,1 \%(2)$ & $11,1 \%(2)$ & $77,8 \%(14)$ & $4,7(0,7)$ \\
\hline Utilidad de la documentación entregada & 0 & 0 & 0 & $10,0 \%(1)$ & $90,0 \%(9)$ & $4,9(0,3)$ \\
\hline Organización general del curso & 0 & 0 & 0 & $11,1 \%(2)$ & $88,9 \%(16)$ & $4,9(0,3)$ \\
\hline Capacidad de los ponentes para resolver dudas & 0 & 0 & 0 & $16,7 \%(3)$ & $83,3 \% / 15)$ & $4,8(0,4)$ \\
\hline Facilidad de entendimiento & 0 & 0 & $5,9 \%(1)$ & $29,4 \%(5)$ & $64,7 \%(11)$ & $4,6(0,6)$ \\
\hline
\end{tabular}

\section{Valoración de las preguntas 10-11}

\begin{tabular}{|l|c|c|c|}
\hline & Insuficiente & Adecuada & Excesiva \\
\hline Duración general de las charlas & 0 & $100 \%(18)$ & 0 \\
\hline Duración de cada tema & 0 & $100 \%(18)$ & 0 \\
\hline
\end{tabular}

\section{Valoración de las preguntas 12-14}

\begin{tabular}{|l|c|c|}
\hline & Si & No \\
\hline Recomendaría el curso & $100 \%(17)$ & 0 \\
\hline La escuela ha resuelto sus dudas e inquietudes & $100 \%(18)$ & 0 \\
\hline Añadiría algún tema & 0 & $33,3 \%(6)$ \\
\hline
\end{tabular}

Tabla 1. Valoración de las preguntas de la encuesta de evaluación 


\section{Discusión y conclusiones}

Entre las distintas estrategias de educación, utilizar el grupo como herramienta, puede presentar ventajas e inconvenientes. Entre las ventajas cabría destacar que las inquietudes y problemas que puedan surgir a un individuo y que a otro aún no se le han presentado, al resolverlas dentro del grupo podrían servir como guía para cuando se le presenten a otros. Por el contrario, siempre puede caber la duda si no puede servir también para inducir problemas en otros que no los sentían. También es bien conocido, que un método de resolver problemas de manera eficaz, en ocasiones muy complejo, es a través de la observación de cómo otros los resuelven, sin precisar un proceso deliberativo racional que lo sustente. Esto también lo aporta el grupo al favorecer la interacción entre personas en un estadio similar de la enfermedad y que se presentan ante la necesidad de planificar cambios inminentes en el estilo de vida propio y de su familia. Además también lo favorecemos con la interacción con pacientes que actúan como mentores explicando su propia experiencia. En general, los pacientes valoran más las experiencias y explicaciones de otros enfermos que viven la experiencia de la enfermedad en primera persona que la del personal sanitario. Es muy útil presentar los testimonios de manera organizada, porque si no los ofrecemos de todas formas los van a buscar y probablemente los encuentren en un lugar inadecuado, de una manera poco estructurada y desorganizada, pudiendo conducir a decisiones basadas en información incorrecta y poco balanceada. Otra de las ventajas que puede presentar el grupo es el tiempo que cada paciente recibe atención, al no ser individualizada, cada uno de los miembros del grupo está expuesto a más de 10 horas de información dirigida a su enfermedad. Nuestros pacientes, aunque de manera preliminar, han demostrado una alta satisfacción con la experiencia de esta intervención. No obstante, la mayoría han experimentado un ligero aumento de ansiedad; lo cual, lo interpretamos como útil en el sentido que les permite movilizarse para cambiar pautas de alimentación, tomar decisiones y probablemente aumentar adherencia la tratamiento. $Y$ desde luego la mayoría parece haber demostrado un aumento de sus conocimientos sobre ERCA y técnicas de sustitución. A más largo plazo, queremos valorar el papel de la intervención sobre variables biológicas como el consumo de fósforo, quelantes del fósforo, potasio, etc,... e incluso, sería interesante valorar si existe un papel protector sobre supervivencia como otros han demostrado ${ }^{8}$, además de incluir en el EMD un trabajador social para abordar aspectos sociales, dada la repercusión que la ERC tiene a nivel laboral y del aumento del grado de dependencia de los pacientes mas añosos.

Recibido: 20 0ctubre 2011

Revisado: 30 Noviembre 2011

Modificado: 27 Diciembre 2011

Aceptado: 18 Enero 2012

\section{Bibliografía}

1. Alcázar R; Orte L; Otero A. Enfermedad renal crónica avanzada. Guías SEN para el manejo de la enfermedad renal crónica avanzada y pre-diálisis. Nefrología 2008; Supl. 3, 3-6.

2. Otero A, de Francisco A, Gayoso P, Garcia F: EPIRCE study Group. Prevalence of chronic renal disease in Spain: results of the EPIRCE study. Nefrología 2010; 30(1):78-86.

3. Marrón $B$, Craver L, Remón $C$, Prieto $M$, Gutierrez $\mathrm{J} \mathrm{M}$, Ortiz A. 'Reality and desire' in the care of advanced chronic kidney disease. NDT plus 2010; 3(5):431-435.

4. Coulter A, Ellins J. Effectiveness of strategies for informing, educating, and involving patients. BMJ 2007; 335(7609):24-27.

5. Low J, Smith G, Burns A and Jones L. The impact of end-stage kidney disease (ESKD) on close persons: a literature review. NDT plus 2008; 1(2):67-79.

6. Levin A, Lewis M, Mortiboy P, Faber S, Hare I, Porter $\mathrm{EC}$ et al. Multidisciplinary predialysis programs: quantification and limitation of their impact on patient outcomes in two Canadian settings. Am J Kidney dis 1997; 29(4):533-540.

7. Spielberger CD, Gorsuch RL, Lushene RE. Inventario de Ansiedad Estado-Rasgo (State-Trait Anxiety Inventory, STAI), Manual STAI/Cuestionario de An- 


\section{[ A. I. Aguilera Flórez, et al ]}

Una estrategia poco utilizada en el cuidado de pacientes con Enfermedad Renal Crónica: la educación en grupo y multidisciplinar de pacientes y sus familiares

siedad Estado-Rasgo, $2^{\mathrm{a}}$ ed. Madrid: TEA Ediciones; 1986.

8. Wu IW, Wang SY, Hsu KH, Lee CC, Sun CY, Tsai CJ et al. Multidisciplinary predialysis education decreases the incidence of dialysis and reduces mortality a controlled cohort study based on the NKF/DOQI guidelines. Nephrol Dial Transplant. 2009; 24(11):34263433. 